\title{
Mit Pestwurz und Musiktherapie gegen kindliche Migräne
}

In der prophylaktischen Behandlung der kindlichen Migräne haben sich nicht medikamentöse Therapieverfahren als besonders wirksam erwiesen. Der genaue Stellenwert von nicht medikamentösen im Vergleich zu medikamentösen Verfahren ist bislang jedoch nur unzureichend untersucht worden. In dieser Studie wurden Kinder und Jugendliche mit Migräne entweder mit Pestwurz, Placebo oder Musiktherapie behandelt. Die wenigsten Attacken hatten die Kinder, die eine Kombination aus Pestwurz und Musiktherapie erhielten.

\begin{abstract}
n der vorliegenden Studie wurden die Kinder und Jugendlichen in drei Gruppen zu je 20 Personen eingeteilt und mit drei verschiedenen Maßnahmen behandelt: mit $100 \mathrm{mg}$ Pestwurz täglich (Petadolex), mit Placebo oder Musiktherapie in einem standardisierten Setting von einer Sitzung pro Woche über insgesamt zwölf Wochen. Zielvariable war die Reduzierung der Kopfschmerzfrequenz über acht Wochen nach der Therapie und über weitere sechs Monate später.
\end{abstract}

\section{Kombiniert am effektivsten}

In der Phase direkt nach der Behandlung sank die Attackenfrequenz in der Musiktherapie- im Vergleich zur Placebo-Gruppe signifikant. In der Evaluation ein halbes Jahr später waren sowohl Musiktherapie als auch Pestwurz im Hinblick auf die Reduzierung der Migräne wirksamer als Placebo. Die Autoren folgern daraus, dass Musiktherapie und möglicherweise die Kombination von Musiktherapie und Pestwurz eine wirksame
Therapie in der Behandlung der kindlichen Migräne darstellt.

Kommentar: Diese Studie zeigt den sehr interessanten und innovativen Ansatz, dass ein standardisiertes nicht medikamentöses Therapieverfahren (hier ein Musiktherapieprogramm über zwölf Wochen) in einem randomisierten Parallelgruppenverfahren mit einem Medikament und mit Placebo verglichen wurde. Obwohl die Patientengruppen klein waren und die Studie daher nur explorativen Charakter hatte, sollte sie doch Anlass bieten, um weitere Studien in diesem Design durchzuführen. Insbesondere unterstützt diese Studie einmal mehr die Erkenntnis, dass nicht medikamentöse Gruppentherapieverfahren in der Behandlung der Migräne bei Kindern und Jugendlichen besonders wirksam sind.

Prof. Dr. Stefan Evers

Oelkers-Ax R et al. Butterbur root extract and music therapy in the prevention of childhood migraine: an explorative study. Eur J Pain 2008; 12: $301-13$

\section{Selbst gewählte Musik beeinflusst Autofahren nicht negativ}

Musikhören ist inzwischen ein fester Umweltbestandteil, typischerweise in privaten Situationen oder großen Kaufhäusern. Ihre Bandbreite reicht dabei vom Belästigungsfaktor bis zur Stimmungsaufhellung. Auch beim Autofahren wird häufig im Hintergrund Musik gehört. In der vorliegenden Studie wurde untersucht, wie Musik die Performance beim Autofahren beeinflusst.

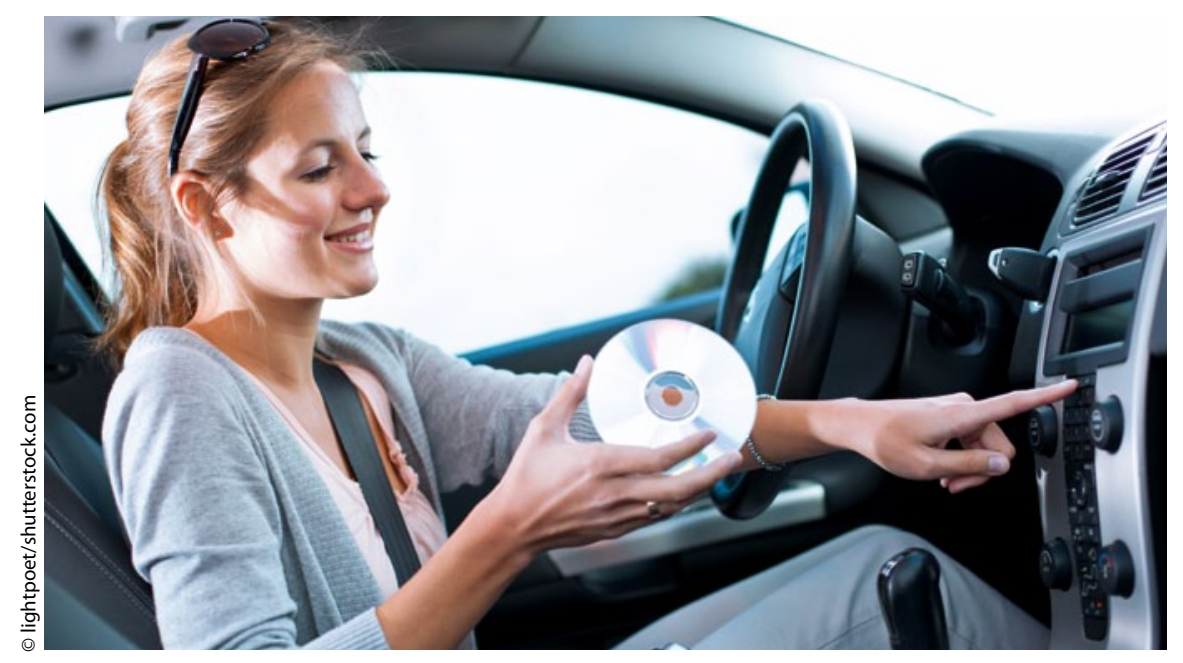

Selbst gewählte Musik hat keine negativen Auswirkungen auf das Fahrverhalten. önnen Stimmungsänderungen, die durch Musik erzielt werden, auch beim Autofahren aufrechterhalten werden? Und verändert sich das Fahrverhalten unter selbst gewählter Musik? Um diese Fragen zu beantworten, simulierten holländische Forscher eine alltägliche Fahrsituation. Die Untersuchungen zeigten, dass sich die durch Musik erzielte Stimmung auch während des Autofahrens aufrechterhält. Es wurde auch deutlich, dass sich in anspruchsvollen Fahrsituationen (insbesondere bei enger Spurbreite) kein Unterschied zwischen Autofahren mit selbst gewählter und ohne Musik ergab. Außerdem war die Respirationsrate während des Musikhörens bei unveränderten kardialen Parametern erniedrigt.

\section{Im Fahrsimulator keine negativen Auswirkungen auf das Fahren}

Die Autoren folgern aus der Studie, dass Hören von selbst gewählter Musik beim Autofahren die Stimmung stabilisieren kann und es nicht zu negativen Auswirkungen auf das Fahrverhalten in anspruchsvollen Situationen kommt. 